\title{
Avaliação de métodos de preparação de amostras de ligante asfáltico para caracterizações microestruturais no microscópio de força atômica
}

\author{
Thaísa Ferreira Macedo ${ }^{1}$, Patrícia Hennig Osmari ${ }^{2}$, Francisco Thiago Sacramento Aragão ${ }^{3}$, \\ Leni Figueiredo Mathias Leite ${ }^{4}$, Renata Antoun Simão ${ }^{5}$
}

\begin{abstract}
1Programa de Engenharia Civil (COPPE) - Universidade Federal do Rio de Janeiro, thaisa_197@hotmail.com 2Programa de Engenharia Civil (COPPE) - Universidade Federal do Rio de Janeiro, patriciaosmari@gmail.com ${ }^{3}$ Programa de Engenharia Civil (COPPE) - Universidade Federal do Rio de Janeiro, fthiago@coc.ufrj.br ${ }^{4}$ Programa de Engenharia Civil (COPPE) - Universidade Federal do Rio de Janeiro, lenimathias@yahoo.com.br ${ }^{5}$ Programa de Engenharia Civil (COPPE) - Universidade Federal do Rio de Janeiro, renata@metalmat.ufrj.br
\end{abstract}

\section{Recebido:}

11 de março de 2018

Aceito para publicação:

17 de julho de 2018

Publicado:

4 de novembro de 2018

Editor de área:

Jorge Barbosa Soares

\section{Palavras-chaves:}

Ligante asfáltico,

Métodos de preparação,

Microscópio de força atômica.

\section{Keywords:}

Asphalt binder,

Preparation methods,

Atomic force microscope.

DOI:10.14295/transportes.v26i3.1616

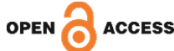

\begin{abstract}
RESUMO
O microscópio de força atômica (AFM) tem sido usado para a caracterização microestrutural de ligantes asfálticos, a partir da identificação das suas frações constituintes. Adicionalmente, estudos mais recentes têm determinado propriedades mecânicas dos componentes dos ligantes, tais como fluência e módulo de relaxação, a partir de ensaios de indentação no AFM. No entanto, o processo de preparação das amostras usadas nestes ensaios geralmente não é descrito detalhadamente na literatura. Este trabalho tem o objetivo de avaliar diferentes métodos de preparação de amostras, empregando imagens topográficas usadas para caracterizações microestruturais. Para tal, microestruturas de um ligante obtidas de amostras preparadas usando diferentes métodos foram avaliadas e os resultados contribuíram para identificar o processo de preparação mais otimizado.
\end{abstract}

\section{ABSTRACT}

The atomic force microscope has been used for the microstructural characterization of asphalt binders from the identification of their constituent fractions. Moreover, recent studies have determined mechanical properties of the binder components, such as creep compliance and relaxation modulus, by indentation tests in the AFM. However, the preparation process of the samples used in these tests is generally not well described in the literature. This study aims to evaluate different preparation methods, employing topography images used for microstructural characterization. For that, microstructures of a binder obtained from samples prepared using different procedures were evaluated and the results contributed to identify the most optimized preparation process.

\section{INTRODUÇÃO}

Nas últimas décadas, o microscópio de força atômica, conhecido no inglês como Atomic Force Microscope (AFM), tem sido usado para investigar a morfologia de ligantes asfálticos (Loeber et al., 1996; Jager et al., 2004; Masson et al., 2006; Zhang et al., 2012). Este equipamento permite a obtenção de imagens microestruturais de alta resolução de filmes asfálticos para a avaliação da topografia e de propriedades mecânicas como módulo de elasticidade, dureza, adesão, entre outras. Tais análises proporcionam um entendimento mais aprofundado sobre as microestruturas e alguns pesquisadores têm avaliado correlações entre os resultados obtidos a partir da análise das imagens topográficas do AFM e demais escalas de investigação como a reológica e a química (Nazzal et al., 2014; Davis e Castorena, 2015; Osmari, 2016; Osmari et al., 2017). 
Até hoje, não há um consenso na literatura sobre a composição microestrutural dos ligantes asfálticos e nem sobre a correlação desta composição com as frações químicas constituintes dos ligantes. Alguns pesquisadores identificam três estruturas nas imagens obtidas no AFM, em função da topografia apresentada por cada uma delas (Davis e Castorena, 2015; Menapace et al., 2015; Veytskin et al., 2015). Estas estruturas são tipicamente denominadas catanaphase, paraphase e periphase. Outros autores consideram ainda a existência da salphase que é formada de pequenas dispersões na paraphase, porém nem sempre são observadas em todas as microestruturas de ligantes (Osmari, 2016).

Além disso, os ligantes costumam apresentar diferentes configurações microestruturais em função da origem do petróleo, da história térmica e do método de preparação da amostra (Yu et $a l ., 2015)$. Enquanto alguns ligantes exibem microestruturas de superfície com domínios finos ou domínios em forma de flocos, outros ligantes apresentam estruturas que se assemelham a abelhas, conhecidas como bees, também chamadas de catanaphase, com padrões ondulados, vários micrômetros de diâmetro e dezenas de nanômetros de altura (Masson et al., 2006).

O estudo realizado por Zhang et al. (2011) também utilizou o AFM para gerar imagens e avaliar características topográficas em escala nanométrica de ligantes antes e após o envelhecimento das amostras. Tais autores afirmaram que por meio do AFM é possível visualizar detalhes precisos de constituintes de ligantes em nanoescala sem o emprego de técnicas especiais de preparação de amostra.

Contudo, estudos complementares ainda são necessários para a definição das complexas características microestruturais dos ligantes asfálticos. Além disso, ensaios de nanoindentação nos filmes de ligantes asfálticos podem ser realizados para a identificação de propriedades mecânicas das diferentes fases constituintes visualizadas pelas microestruturas obtidas em um AFM. Investigações neste sentido, podem contribuir para a determinação de características microestruturais que auxiliam na complementação de estudos de multiescala experimental para a avaliação do comportamento das misturas asfálticas em campo.

A literatura referenciada sobre AFM em ligantes asfálticos descreve diferentes métodos de preparação dos filmes asfálticos para avaliação topográfica. Parâmetros como a espessura do filme, a sua forma de preparação (solução em tolueno, spin coating, heat casting) e a quantidade de amostra necessária para a caracterização da topografia das frações constituintes são significativamente variáveis e nem sempre bem discriminados.

A preparação das amostras de ligantes asfálticos para realização de varreduras no AFM é uma etapa crucial na análise das microestruturas, uma vez que possibilita a identificação de diferentes frações constituintes nos materiais. 0 levantamento bibliográfico revelou que existem vários procedimentos de preparação, tais como o método de fusão em solução, o método de fusão a quente, entre outros. A opção por um método em detrimento de outros tem sido feita de acordo com a preferência do pesquisador, o tipo de ligante, o tempo disponível para preparar a amostra e a espessura do filme asfáltico desejada.

Loeber et al. (1996) estão entre os primeiros pesquisadores a utilizar o AFM e a analisar as microestruturas de ligantes asfálticos. 0 método de preparação de amostras foi o de fusão por calor (heat cast method), que consiste em despejar uma gota de ligante aquecida a $140^{\circ} \mathrm{C}$ sobre um suporte de disco de aço e resfriá-la até $25^{\circ} \mathrm{C}$.

Masson et al. (2006) compararam microestruturas de diversos ligantes asfálticos com as de Pauli et al. (2001) e verificaram que amostras preparadas empregando o heat cast method e o cast method apresentaram resultados diferentes entre si. Com isto, os autores concluíram que o 
método de preparação das amostras tem influência significativa na morfologia superficial dos materiais asfálticos.

O método de fusão em solução (cast method) consiste em empregar tolueno para a diluição do ligante asfáltico, aplicação no substrato de vidro e evaporação do solvente. Este método permite que sejam geradas amostras de forma rápida e não envolve qualquer procedimento de aquecimento. Allen (2010) usou este método em seu estudo. No entanto, para evitar a oxidação rápida do ligante, cada amostra foi armazenada em uma câmara a vácuo, sendo retirada apenas para a realização das varreduras com o AFM. Segundo o autor, a etapa de armazenagem a vácuo demonstrou ser importante para a captação das microestruturas e a sua omissão pode resultar em imagens distintas entre si para o mesmo ligante asfáltico.

Ainda que essa metodologia permita a preparação de amostras sem aquecimento, existem algumas desvantagens, tais como o fato de a amostra ter que permanecer em repouso em local sem luz e umidade, durante uma semana, para permitir que o excesso de tolueno se evapore. 0 tolueno, se presente, pode influenciar as ligações químicas dos ligantes (Mccarron et al., 2012). A espera por sete dias para a evaporação do tolueno também foi citada em trabalhos desenvolvidos na China na visualização de filmes de ligantes asfálticos no AFM (Hou et al., 2017).

Já o método de fusão a quente (heat cast method), em que ligantes asfálticos são aquecidos e aplicados na placa em estado líquido, resulta em uma aparência opaca e uma espessura maior em relação ao cast method. Cada amostra requer cerca de trinta minutos para a preparação, em função dos tempos de aquecimento e resfriamento dos materiais (Mccarron et al., 2012). Após a etapa da preparação, o filme asfáltico é mantido em repouso por tempo determinado pelo pesquisador até que a amostra possa ser varrida pelo AFM.

Aguiar-Moya et al. (2017) prepararam 10 amostras de cada ligante investigado da seguinte maneira: aplicação de uma pequena parte do ligante sobre uma placa do material Si-wafer (silicone-wafer), que foi mantida em estufa a $163^{\circ} \mathrm{C}$ por 4 minutos para garantir que o ligante estivesse líquido o suficiente para ser distribuído uniformemente sobre a placa formando um filme fino. A amostra foi então aquecida novamente na estufa por 2 minutos para garantir que o filme estivesse uniforme. 0 resfriamento das amostras aconteceu naturalmente em temperatura ambiente em um dessecador por um período de $24 \mathrm{~h}$, evitando a contaminação com materiais em suspensão no ar. A espessura resultante das amostras preparadas com emprego do Siwafer foi de $1 \mathrm{~mm}$.

Shaopeng et al. (2010) revelaram em sua pesquisa que amostras irradiadas por UV apresentaram um aumento da viscosidade. Este efeito foi fortemente dependente da espessura da película exposta de ligante, particularmente quando esta for inferior a $150 \mu \mathrm{m}$. Isso indica que a espessura da amostra de ligante e o método de preparação devem ser avaliados e definidos com bastante cuidado para a obtenção de microestruturas representativas.

O estudo realizado por Pauli et al. (2011) avaliou diferentes espessuras de amostras de filmes asfálticos. Nele, os autores afirmaram que amostras de ligantes com espessuras superiores a 10 $\mu \mathrm{m}$ apresentam microestruturas semelhantes entre si. Já espessuras inferiores a $10 \mu \mathrm{m}$, como, por exemplo, 0,01 $\mu \mathrm{m}, 0,1 \mu \mathrm{m}$ e $1 \mu \mathrm{m}$, apresentaram diferenças significativas nas microestruturas das amostras dos mesmos ligantes asfálticos. Logo, as amostras devem ser produzidas com espessuras superiores a $10 \mu \mathrm{m}$.

Nahar et al. (2013) avaliaram métodos de preparação e acondicionamento de ligantes em laboratórios distintos para a obtenção de imagens no AFM. Os autores observaram uma boa reprodutibilidade das medidas de características microestruturais, o que indica que os métodos 
podem ser comparáveis. Além disso, os autores concluíram que a influência da temperatura no processo de fabricação das amostras é relevante para a obtenção de microestruturas similares nos diferentes laboratórios.

Este estudo tem como objetivo avaliar diferentes procedimentos expeditos de preparação de amostras para a geração de imagens de topografia, verificando o efeito da temperatura, do solvente, do spin coater, do tipo de placa (substrato), da espessura do filme de ligante e da quantidade de ligante em cada substrato para a obtenção de microestruturas no AFM. A preparação deve produzir filmes de ligante que sejam lisos, homogêneos e uniformes, para tornar possível a geração de imagens no AFM. Assim, foi observada a necessidade de investigar diferentes formas de preparação de amostras com o intuito de verificar a mais adequada para análise morfológica dos pontos de vista quantitativo e qualitativo.

\section{MATERIAIS E EQUIPAMENTOS}

O material usado nas caracterizações microestruturais foi um ligante asfáltico classificado, por penetração, como CAP 50/70 e proveniente da Refinaria Duque de Caxias (Reduc).

As microestruturas de filmes asfálticos foram obtidas em um equipamento de AFM Modelo JPK Nano Wizard 1.0, seguindo os procedimentos recomendados por estudos (Backx et al., 2014; Dourado et al., 2014; Pizzorno et al., 2014). As amostras foram varridas pelo modo de contato intermitente do AFM, a $25^{\circ} \mathrm{C}$, em diversas posições. A ponta usada para a geração de imagens de topografia foi de silício, PPP-NCSTAu da empresa Nanosensors. Esta é uma haste retangular que possui frequência de ressonância próxima a $160 \mathrm{kHz}$ e constante de mola de 7,4 $\mathrm{N} / \mathrm{m}$.

Uma imagem de microscopia eletrônica de varredura da haste obtida em um microscópio JEOL JSM 6460-LV, USA operado a 20 kV com 700x de aumento está apresentada na Figura 1. Cada amostra foi varrida em diferentes áreas quadradas medindo $10 \times 10 \mu \mathrm{m}^{2}$ e outras de $50 \mathrm{x}$ $50 \mu \mathrm{m}^{2}$. Pelo menos 10 imagens foram obtidas para cada procedimento de preparação. As imagens apresentadas nos resultados deste trabalho são representativas destas áreas em pelo menos 2 amostras diferentes produzidas da mesma forma.

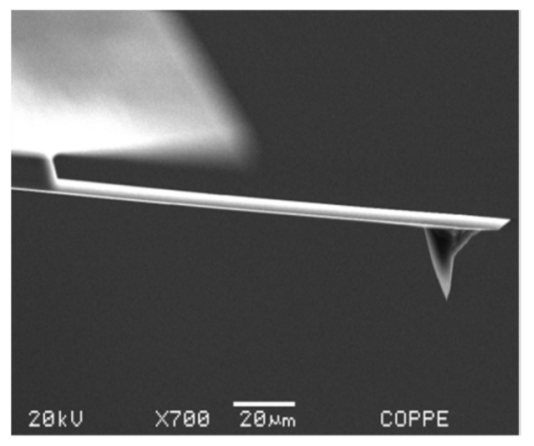

Figura 1: Ponta utilizada no AFM para a geração de imagens do ligante asfáltico obtida em laboratório da COPPE/UFRJ

Além de uma estufa para o aquecimento do ligante, os equipamentos usados na preparação das amostras são ilustrados na Figura 2 e foram os seguintes:

- Spin coater (Figura 2a), para o espalhamento do ligante por meio de rotação. Este equipamento possui acoplamento de mangueira para a aplicação de vácuo, que auxilia na fixação do substrato durante a rotação; 
- Termômetro digital com mira a laser infravermelho MT-320 Minipa (Figura 2b) para permitir a leitura da temperatura sem o contato com o ligante.

\section{MÉTODOS}

Foram definidas, com base na literatura, as seguintes formas de preparação de amostras:

- $1^{\circ}$ ) Ligantes aquecidos em estufa a $120^{\circ} \mathrm{C}$ e colocados sobre placas de alumínio com diferentes espessuras, sem usar o spin coater;

- $2^{\circ}$ ) Uso de placas de vidro com ligante diluído no tolueno, seguido do uso do spin coater para o espalhamento do material sobre a placa;

- $\quad 3^{\text {o}}$ ) Uso de placas de teflon como substrato, aplicando o spin coater;

- $4^{\circ}$ ) Uso de placas de Si-wafer bastante delicadas, sem o uso do spin coater;

- $5^{\circ}$ ) Ligantes aquecidos em estufa a $140^{\circ} \mathrm{C}$ e colocados sobre placas de vidro com diferentes espessuras, usando o spin coater;

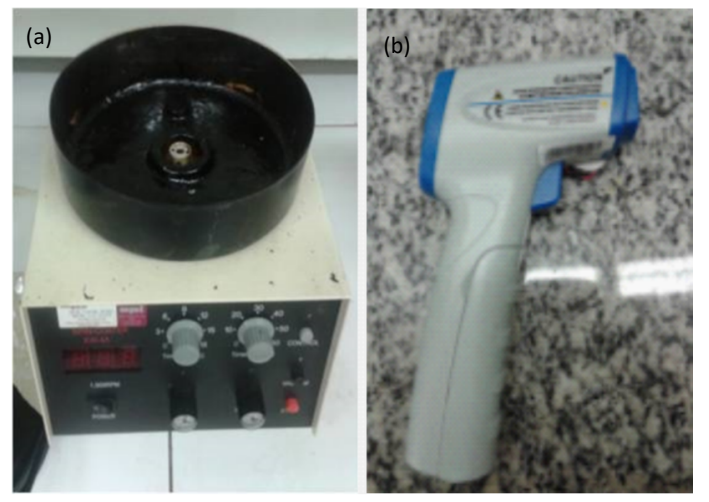

Figura 2: Equipamentos: (a) spin coater e (b) termômetro de infravermelho

O critério de escolha das diferentes metodologias levou em conta quatro fatores, de acordo com a Tabela 1: a espessura, a temperatura de produção, o tipo de resfriamento e o método de produção. A temperatura de $140^{\circ} \mathrm{C}$, no último procedimento (quinto da metodologia), difere dos outros métodos, sendo o mesmo adotado no trabalho de Osmari (2016). Acima de $120^{\circ} \mathrm{C}$, o cimento asfáltico CAP 50-70 possui comportamento newtoniano e não apresenta mais associações moleculares que possam interferir na imagem topográfica do presente estudo.

Tabela 1: Dados referentes ao uso de diferentes substratos e metodologias adotadas

\begin{tabular}{lllll}
\hline Substrato & Espessura $(\mathrm{mm})$ & Temperatura $\left({ }^{\circ} \mathrm{C}\right)$ & Tipo de Resfriamento & Método \\
\hline Alumínio & 0,330 & 120 & Rápido & Heat-casting \\
Alumínio & 0,383 & 120 & Rápido & Heat-casting \\
Teflon & 0,4 & 120 & Lento & Spin-coating \\
Vidro & $0,305-0,334$ & 140 & Rápido & Spin-coating \\
Silício & 0,4 & 120 & Rápido & Heat-Casting \\
Tolueno & $<0,01$ & ambiente & - & Spin-coating \\
\hline
\end{tabular}

\subsection{Procedimento de preparação com placas de alumínio}

As placas de alumínio foram fabricadas com espessuras diferentes com o intuito de verificar o efeito da espessura na microestrutura do ligante com o uso do AFM. A Tabela 2 mostra informa- 
ções relevantes sobre as amostras. 0 material alumínio foi escolhido pois permite rápida transferência de calor por ocasião do seu resfriamento com a placa de mármore. As etapas do procedimento de preparação das amostras foram as seguintes:

- $\quad 1^{\circ}$ ) Aquecer as placas de alumínio para a preparação das amostras;

- $\quad 2^{\text {o}}$ Colocar o ligante nos substratos de alumínio;

- $3^{\circ}$ ) Colocar os substratos de alumínio em placa de mármore fria.

Tabela 2: Dados referentes às amostras nas placas de alumínio

\begin{tabular}{lll}
\hline Dados das amostras & Amostra 1 & Amostra 2 \\
\hline Quantidade $(\mathrm{g})$ & 0,144 & 0,168 \\
Espessura $(\mathrm{mm})$ & 0,330 & 0,383 \\
Diâmetro $(\mathrm{cm})$ & 2,32 & 2,32 \\
Raio $(\mathrm{cm})$ & 1,16 & 1,16 \\
Área $\left(\mathrm{cm}^{2}\right)$ & 4,227 & 4,227 \\
Densidade do ligante $\left(\mathrm{g} / \mathrm{cm}^{3}\right)$ & 1,035 & 1,035 \\
\hline
\end{tabular}

Após o procedimento de preparação das amostras, as placas foram mantidas em repouso por 24 horas para a geração de imagens de topografia no AFM. 0 critério utilizado para realizar essa geração de imagens foi baseado na literatura, pois, geralmente, a amostra é mantida em repouso 24 horas após a preparação.

\subsection{Procedimento de preparação com placas de vidro com o uso do tolueno}

Este procedimento, ilustrado na Figura 3, é conhecido como cast method (método de fusão em solução) e foi baseado na literatura (Allen, 2010; McCarron et al., 2012). 0 método permite a geração de amostras de forma rápida e não envolve aquecimento. Os passos para a obtenção das amostras foram os seguintes:

- $1^{\text {o}}$ ) Colocar $11 \mathrm{~mL}$ de tolueno em 1,5 g de ligante para a diluição do ligante e, com o auxílio de uma pipeta, aplicar gotas da solução de tolueno sobre o substrato de vidro, em temperatura ambiente (Figuras 3 a e $3 \mathrm{~b}$ ). A seguir, o substrato foi submetido ao processo de spin coating. Foram aplicadas diferentes quantidades de gotas $(2 \mathrm{~mL}, 3 \mathrm{~mL}, 4 \mathrm{~mL}, 5$ $\mathrm{mL}$ e $6 \mathrm{~mL}$ ) para verificar o efeito da quantidade de solução nas imagens de AFM;

- $2^{\circ}$ ) Gerar e analisar as imagens do AFM após 24 horas de preparação das amostras de ligante. Neste trabalho, a metodologia empregada por McCarron et al. (2012) foi modificada para torná-la mais expedita. Assim, as amostras não foram deixadas por uma semana no dessecador.

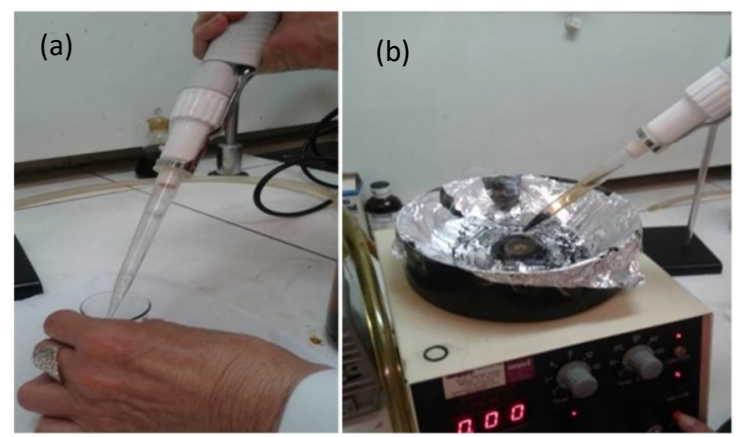

Figura 3: Preparação com solução de tolueno: (a) pipeta para retirar a solução e (b) aplicação das gotas de solução e uso do spin-coater 


\subsection{Procedimento de preparação com placas de teflon}

Foi escolhida a placa de teflon para a preparação de amostras por ser um material flexível e antiaderente, tornando-se mais prática a leitura das espessuras dos ligantes. Então, o ligante foi aquecido em estufa a uma temperatura de $120^{\circ} \mathrm{C}$, de forma a evitar a sua oxidação. 0 ligante foi derramado sobre várias placas de teflon e em seguida estas foram submetidas à rotação com o uso do spin coater. A Figura 4 ilustra amostras em placas de teflon após a rotação.

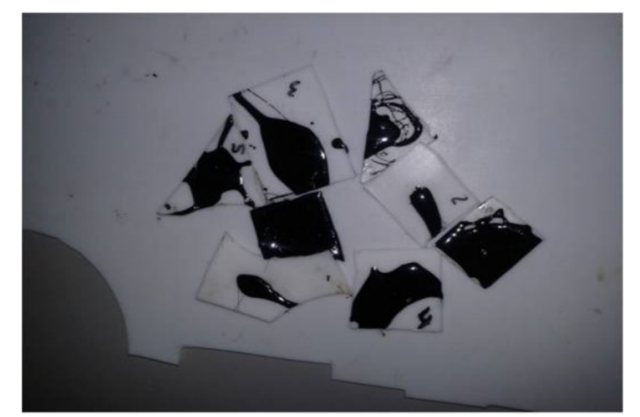

Figura 4: Amostras de ligante em placas de teflon

\subsection{Procedimento de preparação com placas de Si-wafer}

O procedimento de preparação de amostras com placas de Si-wafer foi escolhido com base no estudo realizado por Aguiar-Moya (2017). A placa e a balança estão ilustradas na Figura 5 e o procedimento se baseia nas seguintes etapas:

- 10) Pesar a placa de Si-wafer antes de iniciar o procedimento de ensaio com o objetivo de calcular a espessura do ligante após a cobertura total sobre a placa;

- $2^{o}$ ) Colocar o ligante sobre a placa e passar um jato de nitrogênio sobre a superfície do ligante e pesar posteriormente.

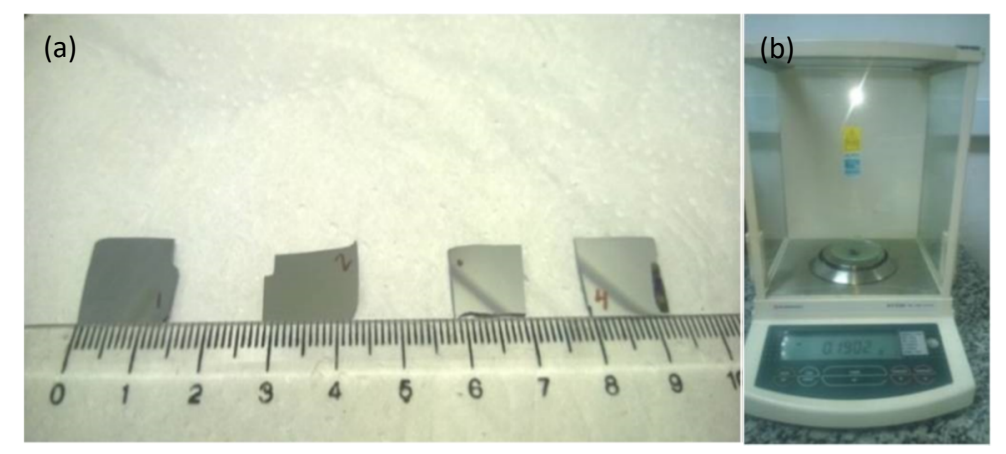

Figura 5: (a) placa de Si-wafer e (b) balança de precisão usadas na preparação de amostras do ligante

\subsection{Procedimento de preparação com placas de vidro e o spin coater}

Baseou-se em vários trabalhos, em que foi introduzido o método heat cast. Este método consiste do aquecimento do ligante e da posterior aplicação de rotação com o uso do spin coater. No trabalho de Osmari (2016), o ligante foi aquecido em estufa a $140^{\circ} \mathrm{C}$ e derramado sobre placas de vidro, previamente limpas, aquecidas e submetidas à rotação, utilizando o spin coater. A Figura 6 apresenta as amostras em placas de vidro após a preparação. As espessuras dessas amostras variaram entre $0,305 \mathrm{~mm}$ e $0,334 \mathrm{~mm}$, sendo valores muito próximos dos obtidos com as placas de alumínio. 


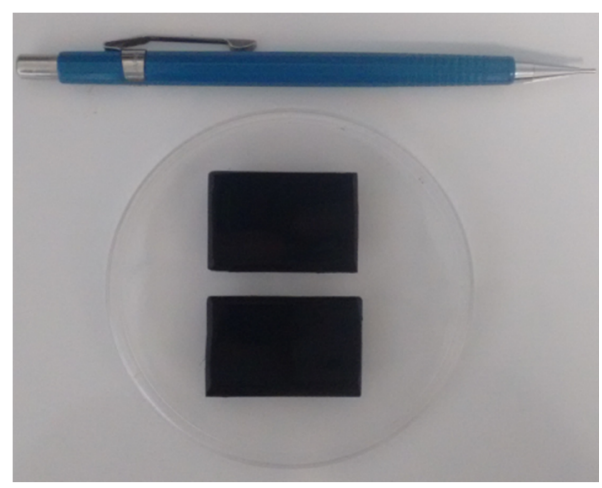

Figura 6: Placas de vidro com o ligante após a preparação (Osmari, 2016)

\section{RESULTADOS}

Optou-se por apresentar as imagens de topografia e somente uma imagem de contraste de fase, pois esta última não acrescenta informação relevante ao que está apresentado na imagem de topografia. As amostras produzidas com placas de alumínio, com diferentes espessuras (330 $\mu \mathrm{m}$ e $383 \mu \mathrm{m}$ ) apresentaram pequenas diferenças nas microestruturas obtidas pelo AFM. A Figura 7 mostra imagens de topografia do ligante sobre as placas de alumínio com $330 \mu$ m e 383 $\mu \mathrm{m}$ de espessura, respectivamente, obtidas pelo AFM sem o uso do spin coater.

A comparação entre essas imagens mostra que a Figura 7(a) apresenta menos estruturas com formatos próximos à catanaphase do que a imagem da Figura 7(b). Estas estruturas podem estar relacionadas às bees que iriam se formar, mas devido ao rápido resfriamento, não se estruturaram completamente. Mesmo resultando em amostras com espessuras superiores a 10 $\mu \mathrm{m}$, o método de preparação com o uso das placas de alumínio não possibilitou a obtenção de microestruturas bem definidas. A catanaphase não se apresentou no formato tradicional de bee. Este resultado pode ser devido ao resfriamento da amostra acontecer de forma muito rápida, a partir do substrato, já que a placa de alumínio era colocada sobre mármore frio imediatamente após ser retirada da estufa. Isto também explica o fato de que o aumento da espessura influencia no número de estruturas tipo bee formadas. Quanto maior a massa de ligante da amostra mais lento o resfriamento, possibilitando a formação de estruturas mais próximas à catanaphase. Assim, tal método não foi considerado o mais adequado. Resultados similares foram obtidos por Pauli et al. (2011) para amostras de $1 \mu \mathrm{m}$ de espessura, para filmes produzidos por spin casting em tolueno.

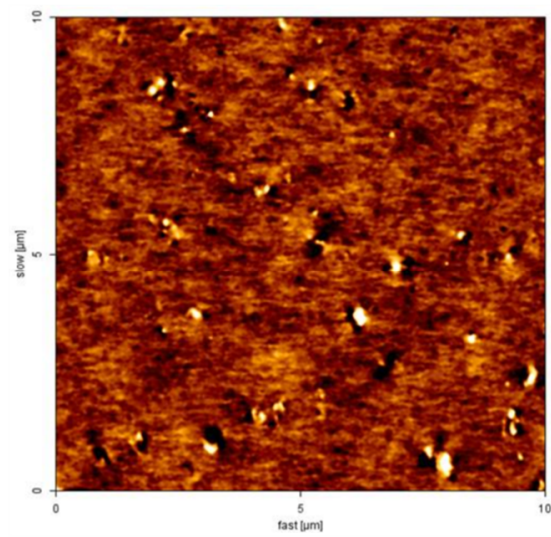

(a)

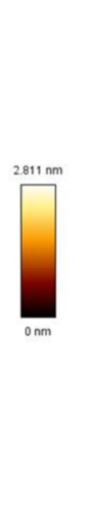

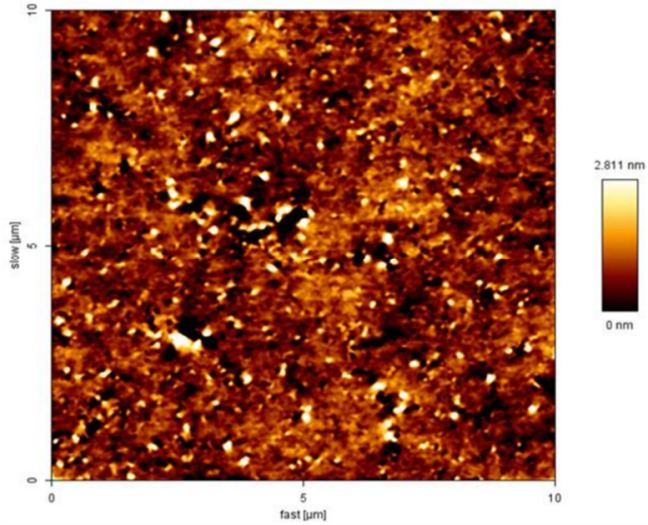

(b) preparação 
As amostras produzidas com as placas de teflon e de Si-wafer, que se resfriariam mais lentamente, não geraram microestruturas apropriadas após as varreduras com o AFM. Esses filmes asfálticos apresentaram irregularidades detectáveis a olho nu na superfície das amostras. Essa característica não é considerada adequada para a obtenção de imagens pelo AFM, uma vez que as superfícies não apresentaram uniformidade e homogeneidade. As placas de teflon são bastante flexíveis, o que implicou em deformações nas placas após rotação do spin coater, aplicação do vácuo e também como consequência da alta temperatura do ligante $\left(120^{\circ} \mathrm{C}\right)$. Desta forma, não foi possível obter espessuras uniformes, tampouco a obtenção das imagens no AFM.

O emprego de placas de vidro com solução de ligante asfáltico e tolueno não gerou microestruturas com o constituinte catanaphase em nenhuma das amostras varridas pelo AFM. A Figura 8 mostra imagens de AFM em que foram usadas 6 gotas de solução para sua preparação com diferentes áreas de captação de dados, 50 x $50 \mu \mathrm{m}^{2}$ (Figura 8a, topografia e 8b, contraste de fase) e $10 \times 10 \mu \mathrm{m}^{2}$ (Figura 8c, topografia). Todas as imagens não apresentaram as bees.

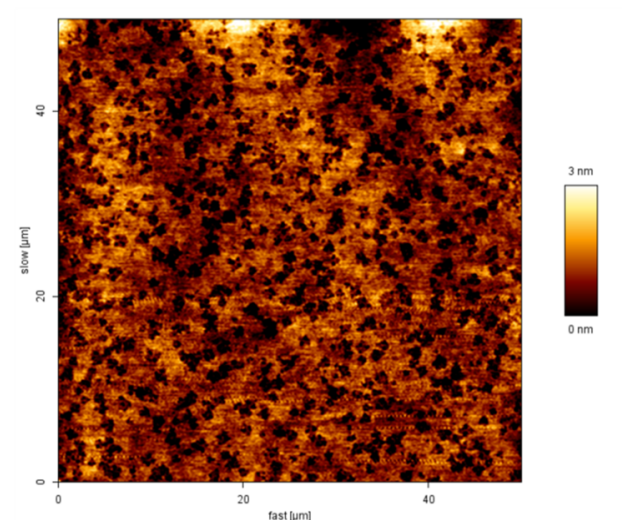

(a)

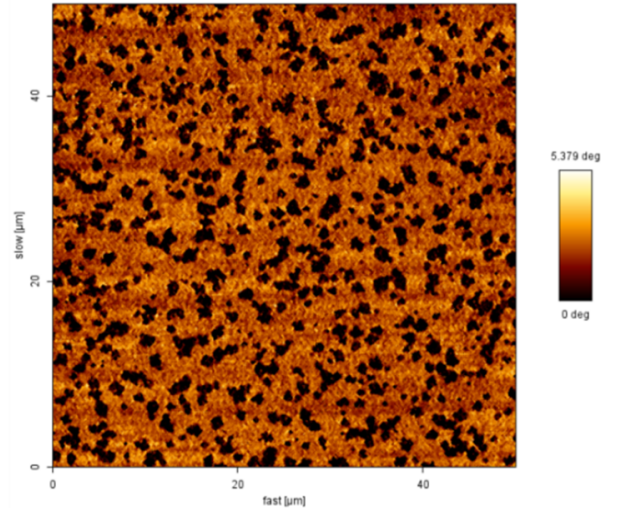

(b)

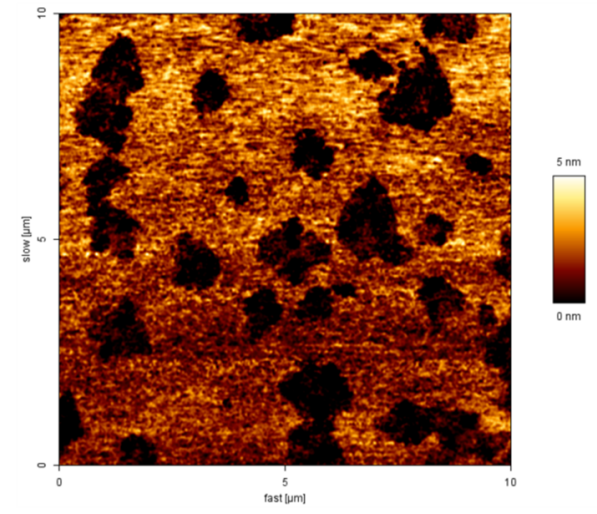

(c)

Figura 8. Amostras produzidas a partir de solução de tolueno: 6 gotas de solução em área de (a) $50 \times 50 \mu m^{2}$ (topografia); (b) $50 \times 50 \mu \mathrm{m}^{2}$ (contraste de fase); (c) $10 \times 10 \mu \mathrm{m}^{2}$ (topografia)

0 fato de se ter empregado o método spin coating em soluções com tolueno para o preparo do filme pode ter sido uma das razões para a não formação da topografia esperada. Na imagem de contraste de fase, nenhuma nova estrutura foi formada. Isto se deve, possivelmente, às pequenas espessuras das amostras, que ficaram extremamente finas após o processo do spin coating. Desse modo, este processo de obtenção de amostras não é indicado para a caracterização das microestruturas usualmente encontradas na literatura. 
A Figura 9 apresenta imagens de topografia de área $50 \times 50 \mu \mathrm{m}^{2}$ das amostras produzidas a partir da solução de tolueno, com diferentes quantidades de gotas. As imagens das Figuras 9(a), 9(b) e 9(c) apresentaram microestruturas semelhantes entre si com a presença de rebaixamentos (formações "circulares" na cor preta), enquanto que a imagem da Figura 9(d) apresentou uma microestrutura bastante diferenciada em relação às demais, possivelmente em função da menor quantidade de solução empregada na moldagem da amostra, uma vez que, no fundo da imagem, a mesma microestrutura foi observada. Além disso, em nenhuma das imagens foi observada a formação de catanaphase e periphase, mostrando que tal método não apresenta uma caracterização microestrutural tradicional de ligantes convencionais. Esta microestrutura convencional representa a imagem do ligante com as quatro fases (catanaphase, periphase, paraphase e salphase).

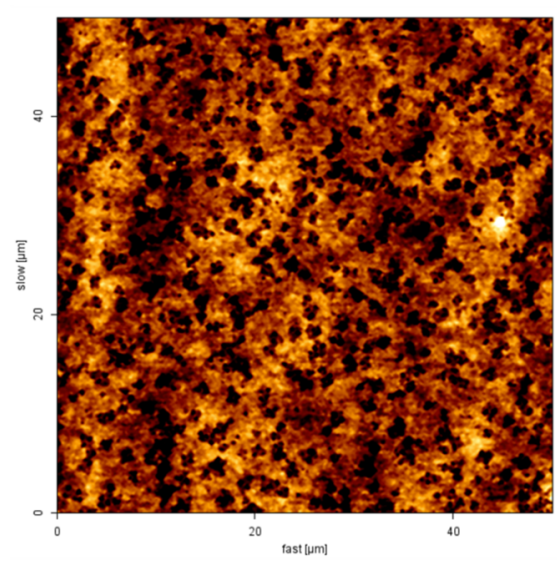

(a)

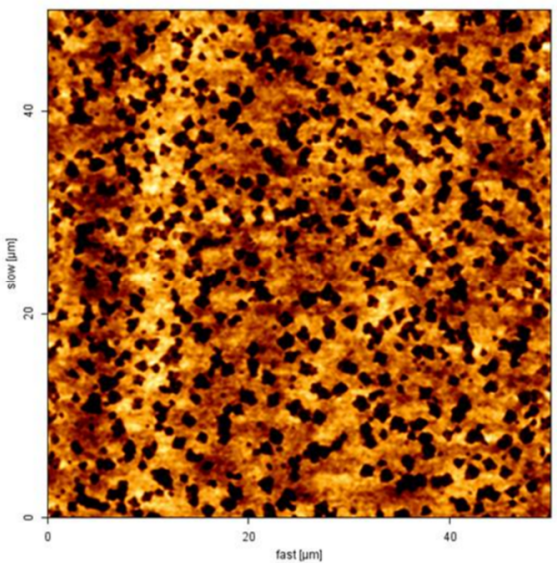

(c)
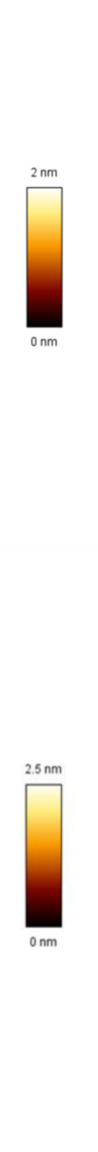

Figura 9. Imagens de topografia: de solução $\left(50 \times 50 \mu \mathrm{m}^{2}\right)$ e (d) 2 gotas de solução $\left(50 \times 50 \mu \mathrm{m}^{2}\right)$

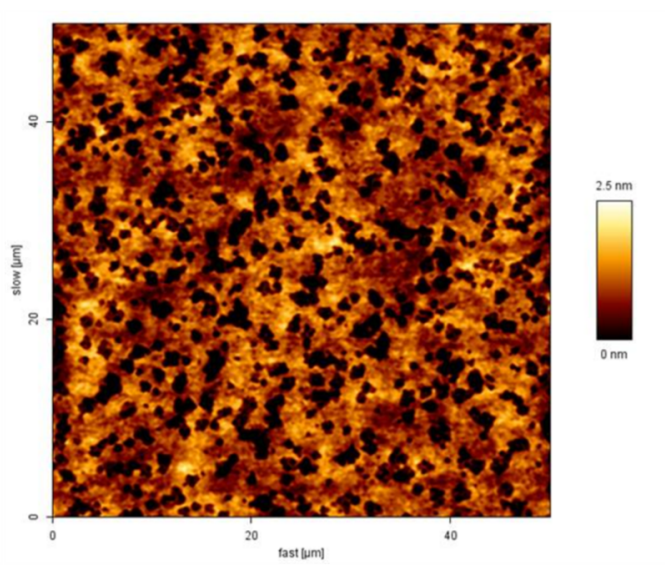

(b)

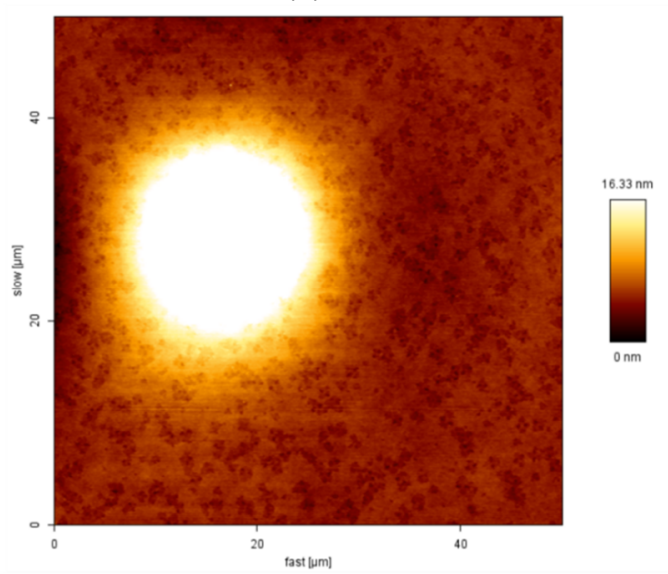

(d)

Por outro lado, as amostras que foram aquecidas em estufa a $140^{\circ} \mathrm{C}$, submetidas ao heat cast method, colocadas sobre placas de vidro e rotacionadas utilizando o spin coater, foram as que apresentaram a maioria das microestruturas (catanaphase, periphase e paraphase) já reportadas na literatura (Davis e Castorena, 2015; Menapace et al., 2015; Veytskin et al., 2015), conforme a Figura 10. As espessuras dessas amostras fabricadas com as placas de vidro $(0,305 \mathrm{~mm}$ e 0,334 mm), foram semelhantes às espessuras das amostras fabricadas com as placas de alumínio $(0,330 \mathrm{~mm}$ e $0,383 \mathrm{~mm})$. Contudo, apresentaram diferenças significativas nas imagens 
microestruturais (Figuras 7 e 10), provavelmente devido ao uso do spin coater para o resfriamento e o espalhamento das amostras nas placas de vidro. Neste caso, o resfriamento ocorreu de forma rápida, mas mais lentamente do que o resfriamento no substrato de alumínio.

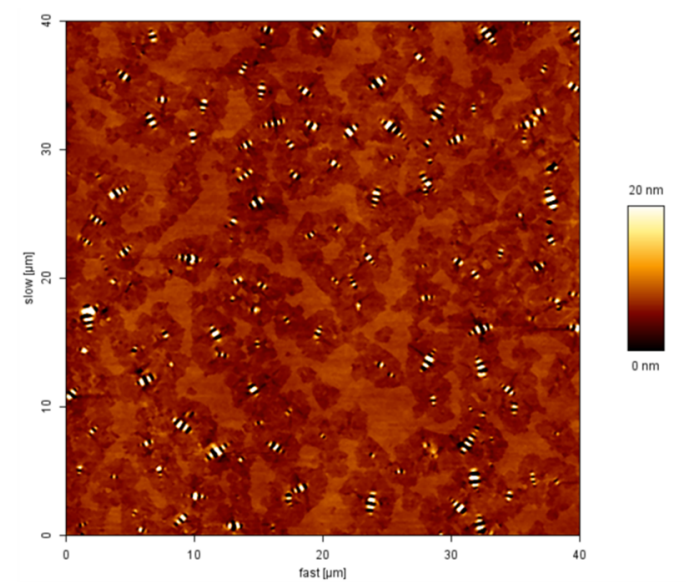

Figura 10. Imagem das microestruturas do ligante CAP 50-70 virgem (50 x $\left.50 \mu \mathrm{m}^{2}\right)$ obtida no AFM com o ligante que foi aquecido a $140^{\circ} \mathrm{C}$ e rotacionado no spin coater (Osmari, 2016)

\section{CONSIDERAÇÕES FINAIS}

A partir da avaliação de diferentes métodos expeditos de preparação de amostras para ensaios no microscópio de força atômica, pode-se concluir que:

- Não foi possível obter imagens no AFM para as amostras produzidas com as placas de teflon e com o Si-wafer. Isto se deve às irregularidades na superfície das amostras, que não ficaram lisas, homogêneas e uniformes;

- As amostras produzidas em placas de alumínio apresentaram pequenas diferenças nas imagens de AFM. As espessuras destas amostras foram 0,33 $\mathrm{mm}$ e 0,38 $\mathrm{mm}$, acima do limite mínimo proposto por Pauli et al. (2011). No entanto, as microestruturas obtidas não se apresentaram bem definidas e, portanto, não foram efetivas para a obtenção de imagens contendo as quatro fases (paraphase, periphase, catanaphase e salphase);

- A estrutura de catanaphase pode não ter sido observada nas amostras produzidas em placas de vidro com a solução em tolueno por conta do processo de spin coating para a formação do filme, pois a evaporação mais rápida do tolueno devido à centrifugação e à formação de filme de pequena espessura afetou a organização estrutural do ligante.

- A amostra produzida pelo heat cast method, que consiste em aquecer o ligante e empregar o spin coater, apresentou espessura próxima às obtidas nas placas de alumínio, acima de 0,010 mm (valor mínimo de espessura), com microestrutura muito bem definida pela imagem gerada no AFM, similar às encontradas na literatura.

A partir destas considerações, pode-se concluir que o método expedito mais apropriado para a caracterização qualitativa de microestruturas de ligantes por meio de topografia com o uso do AFM é o heat cast method associado ao emprego do spin coater.

\section{AGRADECIMENTOS}

Os autores agradecem ao Conselho Nacional de Desenvolvimento Científico e Tecnológico (CNPq) pelas bolsas de produtividade em pesquisa e de doutorado, à Coordenação de Aperfeiçoamento de Pessoal de Nível Superior (CAPES) pela bolsa de doutorado e à Fundação Carlos Chagas Filho de Amparo à Pesquisa do Estado do Rio de Janeiro (FAPERJ) pela bolsa de jovem cientista do nosso estado. 


\section{REFERÊNCIAS}

Aguiar-Moya, J. P.; J. Salazar-Delgado; A. García; A. Baldi-Sevilla; V. Bonilla-Mora e L. G. Lória-Salazar (2017) Effect of aging on micromechanical properties of bitumen by means of atomic force microscopy. Road Materials and Pavement Design, v. 18, p. 203-215. DOI: 10.1080/14680629.2017.1304249.

Allen, R. G. (2010). Structural Characterization of Micromechanical Properties in Asphalt Using Atomic Force Microscopy. Dissertação (Mestrado em Engenharia Civil), Texas A\&M, College Station, TX, USA.

Backx, B. P.; R. A. Simão; E. R. Dourado e L. F. M. Leite (2014) Solvent Effect on the Morphology of the Bee - Structure Observed by Atomic Force Microscopy on Bitumen Sample. Research Materials, v. 17, p. 1157-1161. D0I: 10.1590/15161439.236113.

Davis, C. e C. Castorena (2015) Implications of physico-chemical interactions in asphalt mastics on asphalt microstructure. Construction and Building Materials, v. 94, p. 83-89. DOI: 10.1016/j.conbuildmat.2015.06.026.

Dourado, E. R.; B. S. Pizzorno; L. M. G. Motta; R. A. Simão e L. F. M. Leite (2014) Analysis of asphaltic binders modified with PPA by surface techniques. Journal of Microscopy, v. 254, p. 122-128. DOI: 10.1111/jmi.12123.

Hou, Y.; L. Wang; D. Wang; M. Guo; P. Liu e J. Yu (2017) Characterization of Bitumen Micro-Mechanical Behaviors Using AFM, Phase Dynamics Theory and MD Simulation. Materials, v. 10, p.1-16. DOI: 10.3390/ma10020208.

Jager, A.; R. Lackner; C. Eisenmenger-Sittner e R. Blab (2004) Identification of Microstructural Components of Bitumen by Means of Atomic Force Microscopy (AFM). Proceedings in Applied Mathematics and Mechanics, v. 4, p. 400-401. DOI: 10.1002/pamm.200410181.

Loeber, L; O. Sutton; J. Morel; J. M. Valleton e G. Muller (1996) New Direct Observations of Asphalts and Asphalt Binders by Scanning Electron Microscopy and Atomic Force Microscopy. Journal of. Microscopy, v. 182, p. 32-39. DOI: 10.1046/j.13652818.1996.134416.x.

Masson, J. F.; V. Leblond, e J. Margeson (2006) Bitumen morphologies by phase-detection atomic force microscopy. Journal of Microscopy, v. 221, p. 17-29. DOI: 10.1111/j.1365-2818.2006.01540.x.

Mccarron, B.; X. Yu; M. Tao e N. Burnham (2012) The Investigation of "Bee -Structures" in Asphalt Binders. Projeto de Qualificação, Worcester Polytechnic Institute: Worcester, MA, USA.

Menapace, I.; E. Masad; A. Bhasin e D. Little (2015) Microstructural properties of warm mix asphalt before and after laboratory simulated long-term ageing. Road Materials and Pavement Design, v. 16, p. 2-20. DOI: 10.1080/14680629.2015.1029692.

Nahar, S. N.; B. Dillingh; S. Erkens; A. J. M. Schmets; H. R. Fischer; A. Scarpas e G. Schitter (2013) Is atomic force microscopy suited as tool for fast screening of bituminous metrials? An interlaboratory comparision study. 92nd Annual Meeting of the Transportation of Research Board, Washington, D. C., USA.

Nazzal, M. D.; W. Mogawer; S. Kaya e T. Bennert (2013) Nanoscale Evaluation of Effect of RAP on Virgin Asphalt Binder Properties. 92th Transportation Research Board Annual Meeting, 13-0153, Washington, D. C., USA.

Osmari, P. H. (2016) Caracterização química, reológica e de morfologia superficial de ligantes asfálticos modificados por diferentes agentes rejuvenescedores. Dissertação (Mestrado em Engenharia Civil), COPPE, Universidade Federal do Rio de Janeiro, Rio de Janeiro, RJ, Brasil.

Osmari, P. H.; F. T. S. Aragão; F. M. L. Leite; R. A. Simão; L. M. G. Motta e Y. R. Kim (2017) Chemical, Microstructural, and Rheological Characterizations of Binders to Evaluate Aging and Rejuvenation. Transportation Research Record: Journal of the Transportation Research Board, n. 2632, TRB, National Research Council, Washington, D.C. DOI: 10.3141/2632-02.

Pauli, A.T.; J. F. Branthaver; R. E. Robertson e W. Grimes (2001) Atomic force microscopy investigation of SHRP asphalts. Symposium on Heavy Oil and Resid Compatibility and Stability, Petroleum Chemistry Division, American Chemical Society, p. 110-114., San Diego, California.

Pauli, A. T.; R. W. Grimes; A. G. Beemer; T. F. Turner e J. F. Branthaver (2011) Morphology of asphalts, asphalt fractions and model wax-doped asphalts studied by atomic force microscopy. International Journal of Pavement Engineering, v. 12, p. 291-309. DOI: 10.1080/10298436.2011.575942.

Pizzorno, B. S.; E. R. Dourado; M. B. De Moraes; R. A. Simão e L. F. M. Leite (2014) Segregation and crystallization of waxes on the surface of asphalt binders as observed by atomic force microscopy. Petroleum Science and Technology, v. 32, pp. 27382745. DOI: 10.1080/10916466.2014.887099.

Shaopeng, W.; P. Ling; L. Gang e Z. Jiqing (2010) Laboratory study on ultraviolet radiation aging of bitumen. Journal of Materials in Civil Engineering, v. 22, p. 767-772. DOI: 10.1061/(ASCE)MT.1943-5533.0000010.

Veytskin, Y.; C. Bobko e C. Castorena (2015) Nanoindentation investigation of asphalt binder and mastic cohesion. Construction and Building Materials, v. 28, p. 163-171. DOI: 10.1016/j.conbuildmat.2015.09.053.

Zhang, H. L.; J. Y. Yu; Z. G. Feng; L. H. Xue e S. P. Wu (2012) Effect of aging on the morphology of bitumen by atomic force microscopy. Journal of Microscopy, v. 246, p. 11-19. DOI: 10.1111/j.1365-2818.2011.03578.x.

Yu Xiaokong, B. N. A. e M. Tao (2015) Surface microstructure of bitumen characterized by atomic force microscopy, Advances in Colloid and Interface Science, v. 218, pp. 17-33. DOI: 10.1016/j.cis.2015.01.003. 